converts T-cell autoreactivity into overt autoimmune disease. Nat. Med. 11:138-145.

10. Devendra, D., et al. 2005. Interferon-alpha as a mediator of polyinosinic:polycytidylic acid-induced type 1 diabetes. Diabetes. 54:2549-2556.

11. Sobel, D.O., et al. 1998. Low dose poly I:C prevents diabetes in the diabetes prone BB rat. J. Autoimmun. 11:343-352.

12. Sobel, D.O., and Ahvazi, B. 1998. Alpha-interferon inhibits the development of diabetes in NOD mice. Diabetes. 47:1867-1872.

13. Rifkin, I.R., Leadbetter, E.A., Busconi, L., Viglianti, G., and Marshak-Rothstein, A. 2005. Toll-like receptors, endogenous ligands, and systemic autoimmune disease. Immunol. Rev. 204:27-42.

14. Bennett, L., et al. 2003. Interferon and granulopoiesis signatures in systemic lupus erythematosus blood. J. Exp. Med. 197:711-723.

15. Baechler, E.C., et al. 2003. Interferon-inducible gene expression signature in peripheral blood cells of patients with severe lupus. Proc. Natl. Acad. Sci.U.S. A 100:2610-2615.
16. Krause, I., Valesini, G., Scrivo, R., and Shoenfeld, Y. 2003. Autoimmune aspects of cytokine and anticytokine therapies. Am. J. Med. 115:390-397.

17. Kono, D.H., Baccala, R., and Theofilopoulos, A.N 2003. Inhibition of lupus by genetic alteration of the interferon-alpha/beta receptor. Autoimmunity. 36:503-510.

18. Theofilopoulos, A.N., Baccala, R., Beutler, B., and Kono, D.H. 2005. Type I interferons (alpha/beta) in immunity and autoimmunity. Annu. Rev. Immunol. 23:307-336.

19. Frigerio, S., et al. 2002. Beta cells are responsible for CXCR3-mediated T-cell infiltration in insulitis. Nat. Med. 8:1414-1420.

20. Shiow, L.R., et al. 2006. CD69 acts downstream of interferon-alpha/beta to inhibit S1P1 and lymphocyte egress from lymphoid organs. Nature. 440:540-544

21. Le Bon, A., et al. 2003. Cross-priming of CD8+ T cells stimulated by virus-induced type I interferon. Nat. Immunol. 4:1009-1015.

22. Scarim, A.L., et al. 2001. Mechanisms of beta-cell death in response to double-stranded (ds) RNA and interferon-gamma: dsRNA-dependent protein kinase apoptosis and nitric oxide-dependent necrosis. Am. J. Pathol. 159:273-283.

23. Kato, H., et al. 2006. Differential roles of MDA5 and RIG-I helicases in the recognition of RNA viruses. Nature. 441:101-105.

24. Gitlin, L., et al. 2006. Essential role of mda-5 in type I IFN responses to polyriboinosinic:polyribocytidylic acid and encephalomyocarditis picornavirus. Proc. Natl. Acad. Sci. U. S. A. 103:8459-8464.

25. Smyth, D.J., et al. 2006. A genome-wide association study of nonsynonymous SNPs identifies a type 1 diabetes locus in the interferon-induced helicase (IFIH1) region. Nat. Genet. 38:617-619.

26. Ronnblom, L., and Alm, G.V. 2003. Systemic lupus erythematosus and the type I interferon system. Arthritis Res. Ther. 5:68-75.

27. Jahnsen, F.L., Farkas, L., Lund-Johansen, F., and Brandtzaeg, P. 2002. Involvement of plasmacytoid dendritic cells in human diseases. Hum. Immunol. 63:1201-1205.

\title{
Cholesterol precursors and facial clefting
}

\author{
Forbes D. Porter
}

Heritable Disorders Branch, National Institute of Child Health and Human Development, NIH, Department of Health and Human Services, Bethesda, Maryland, USA.

\begin{abstract}
Inborn errors of cholesterol synthesis cause human malformation syndromes, including Smith-Lemli-Opitz syndrome, lathosterolosis, desmosterolosis, X-linked dominant chondrodysplasia punctata type 2, and congenital hemidysplasia with ichthyosiform erythroderma and limb defects. Because adequate cholesterol is not transported across the placenta, low cholesterol and elevated sterol precursor levels are present during embryogenesis. It has been debated whether the malformations result from low cholesterol or the buildup of sterol precursors. In this issue of the JCI, Engelking et al. provide evidence that sterol precursor accumulation plays a pivotal role in the genesis of facial malformations (see the related article beginning on page 2356).
\end{abstract}

\section{Inborn errors of cholesterol synthesis}

Smith-Lemli-Opitz syndrome (SLOS; see ref. 1) is due to mutation of the gene coding for 7-dehydrocholesterol reductase (DHCR7). DHCR7 reduces the $\Delta^{7}$ double bond in 7-dehydrocholesterol (7-DHC) to yield cholesterol in the final step of cholesterol synthesis. Impaired DHCR7 activity causes an accumulation of 7-DHC and cholesterol deficiency. Clinical manifestations of SLOS (Figure 1) are variable, and the phenotypic spectrum is broad. Severely

Nonstandard abbreviations used: 7-DHC, 7-dehydrocholesterol; DHCR7, 7-dehydrocholesterol reductase; DKO, double-knockout; Ptch, Patched; Shh, Sonic hedgehog; SLOS, Smith-Lemli-Opitz syndrome; Smo, Smoothened.

Conflict of interest: The author has declared that no conflict of interest exists.

Citation for this article: $J$. Clin. Invest. 116:2322-2325 (2006). doi:10.1172/JCI29872. affected infants have multiple congenital anomalies and devastating neurological impairment. In contrast, mild SLOS combines learning and autistic-like behavioral problems with minor physical anomalies. Typical craniofacial findings include microcephaly; ptosis; a short, upturned nose; and micrognathia. Cleft palate occurs in approximately half of the patients. Limb anomalies include short thumbs, postaxial polydactyly, and 2-3 toe syndactyly. SLOS is a relatively common genetic disorder. The clinical incidence has been estimated to be on the order of 1 in 20,000 to 1 in 60,000 . However, the carrier frequency for SLOS mutations suggests an incidence on par with more well-known genetic disorders, such as phenylketonuria ( 1 in 14,000) and osteogenesis imperfecta ( 1 in 20,000).

SLOS was identified as a defect of cholesterol synthesis in 1993 (2). Subsequently, 4 additional human malformation syn- dromes have been shown to be due to impaired cholesterol synthesis (reviewed in ref. 3). These include the "SLOS-like" syndromes desmosterolosis and lathosterolosis, which are due to deficiencies of $3 \beta$-hydroxysterol- $\Delta^{24}$-reductase and lathosterol $\Delta^{5}$-desaturase, respectively. Two skeletal dysplasia syndromes with dermatologic manifestations, X-linked dominant chondrodysplasia punctata type 2 and congenital hemidysplasia with ichthyosiform erythroderma and limb defects, result from deficiency of $3 \beta$-hydroxysterol$\Delta^{8}, \Delta^{7}$-sterol isomerase and NADPH sterol dehydrogenase, respectively. Hydrops-ectopic calcification-moth-eaten dysplasia and some cases of Antley-Bixler syndrome may include a minor impairment of cholesterol synthesis (3). However, the contribution, if any, of impaired cholesterol synthesis to these latter 2 syndromes is not clear.

\section{Cholesterol deficiency and precursor toxicity}

Understanding the pathophysiological processes that underlie the developmental defects in SLOS is complicated because cholesterol is important in multiple biological processes. Cholesterol is a structural lipid in cellular membranes and an obligatory biogenic precursor for steroid, oxysterol, and bile acid synthesis, and cholesterol modification of hedgehog morphogenic proteins 

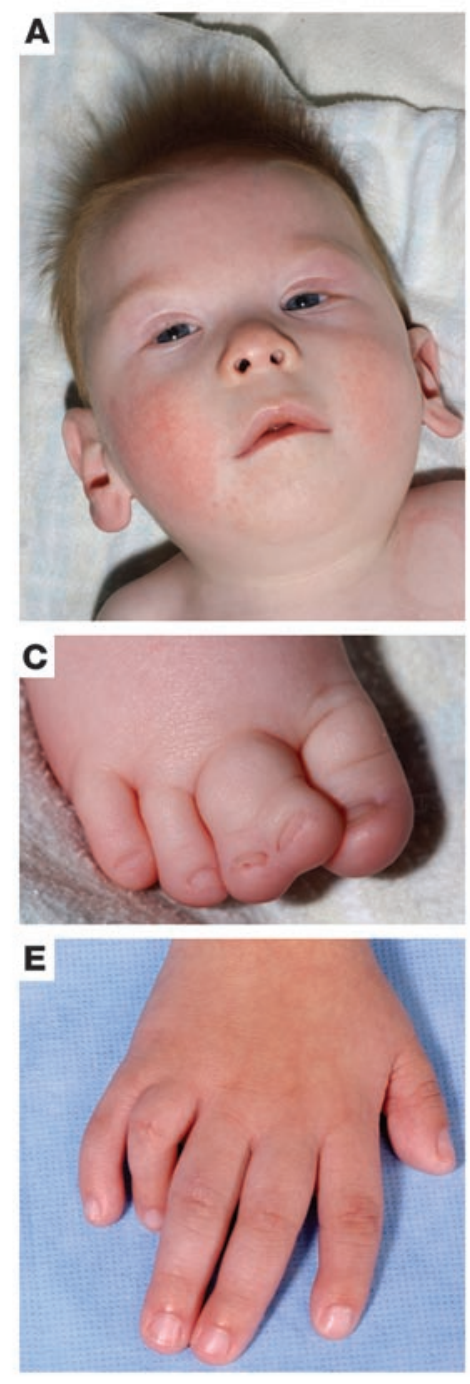
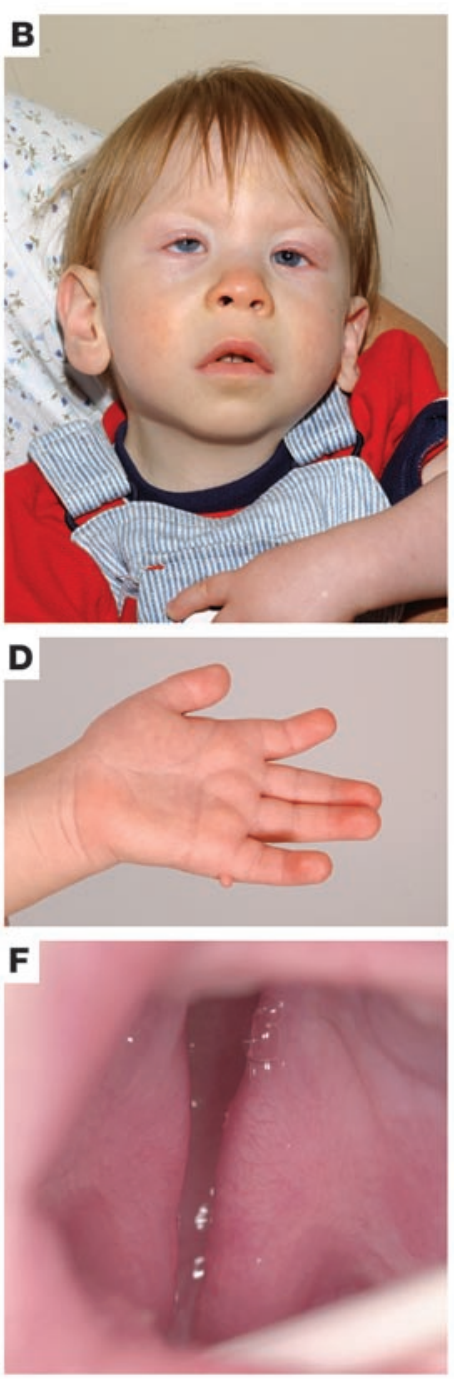

is required for normal hedgehog signaling. Further complicating our understanding of the pathophysiology is that each of these biological processes could be perturbed by either cholesterol deficiency or excessive sterol precursor accumulation.

It has been debated whether the malformations in SLOS and related disorders are due to low cholesterol, low total sterol, or elevated sterol precursor levels. Precursor sterols could be bioactive themselves or give rise to bioactive sterol products, including cytotoxic oxysterols $(4,5)$. The study by Engelking et al. in this issue of the JCI (6) helps separate these factors. This group disrupted both Insig-1 and Insig-2 and studied the developmental consequences in double-knockout (DKO) mice. Insig proteins play a pivotal role in the regulation of cholesterol synthesis by modulating the activity of 2 sterol-sensing domain-containing proteins, Scap and HMG-CoA reductase (reviewed in ref. 7) (Figure 2). When cellular cholesterol levels are low, Scap escorts SREBPs from the endoplasmic reticulum to the Golgi apparatus, where they are activated by proteolytic processing. Activated SREBPs translocate to the nucleus and promote the expression of cholesterologenic genes. When cellular cholesterol levels rise, Insig proteins bind Scap and inhibit the translocation of SREBPs to the Golgi apparatus. Insig proteins also modulate HMG-CoA reductase activity. When the levels of lanosterol, a precursor sterol, increase, Insig binds to and mediates the proteolytic destruction of HMG-CoA reductase.

When both Insig-1 and Insig-2 are disrupted in the liver, cholesterol synthesis becomes dysregulated. Even though cholesterol levels rise significantly above normal, activated SREBPs continue to promote the transcription of cholesterol synthetic genes, and HMG-CoA reductase activity remains

\section{Figure 1}

SLOS phenotype. Typical facial features of a SLOS patient as an infant (A) and young child (B). Cutaneous syndactyly of the second and third toes (C) is the most frequently reported finding in SLOS. Hand anomalies (D and E) may include single palmar creases, short thumbs, and postaxial polydactyly. Some degree of cleft palate $(\mathbf{F})$ is observed in approximately half of the patients.

high (8). Engelking et al. (6) now report the developmental consequences of Insig deficiency. Most Insig-DKO embryos have midline facial clefting ranging from cleft palate to a complete facial cleft. Cleft palate is a malformation found in both humans and mice with inborn errors of cholesterol synthesis. Mouse models of SLOS (9), lathosterolosis (10), and desmosterolosis (11) have been produced by targeted homologous recombination. All 3 of these mouse models exhibit abnormally low tissue cholesterol levels; however, the accumulating precursors differ. Cleft palate is found in SLOS and lathosterolosis mice at a frequency of $9 \%$ and $88 \%$ respectively, but is not found in desmosterolosis mice. These differences suggest that cleft palate frequency may be dependent on the nature of the accumulating sterol precursor. However, because all of these mouse models have decreased cholesterol levels, one cannot exclude the possibility that low cholesterol combined with the differential abilities of 7-DHC, lathosterol, and desmosterol to substitute for cholester$\mathrm{ol}$ is the critical factor. Insig-DKO embryos accumulate sterol precursors but are not cholesterol deficient. Hence, the Engelking et al. study implicates sterol precursor accumulation, rather than diminished cholesterol or total sterol levels, as a key factor in the genesis of facial malformations.

\section{Sonic hedgehog and cleft palate}

The discovery that cholesterol is necessary for the maturation of hedgehog proteins (12) provided insight into a developmental pathway that is likely perturbed due to inborn errors of cholesterol synthesis. The hedgehog proteins play a major role in multiple developmental fields. Sonic hedgehog (Shh) is involved in palate development, and disruption of Shh causes cleft palate (13). Shh undergoes autocatalytic processing, whereby a molecule of cholesterol becomes covalently attached to its signaling domain (12). Cholesterol modification is necessary for normal Shh signaling (14) (Figure 2). Shh binds to the receptor Patched (Ptch). 


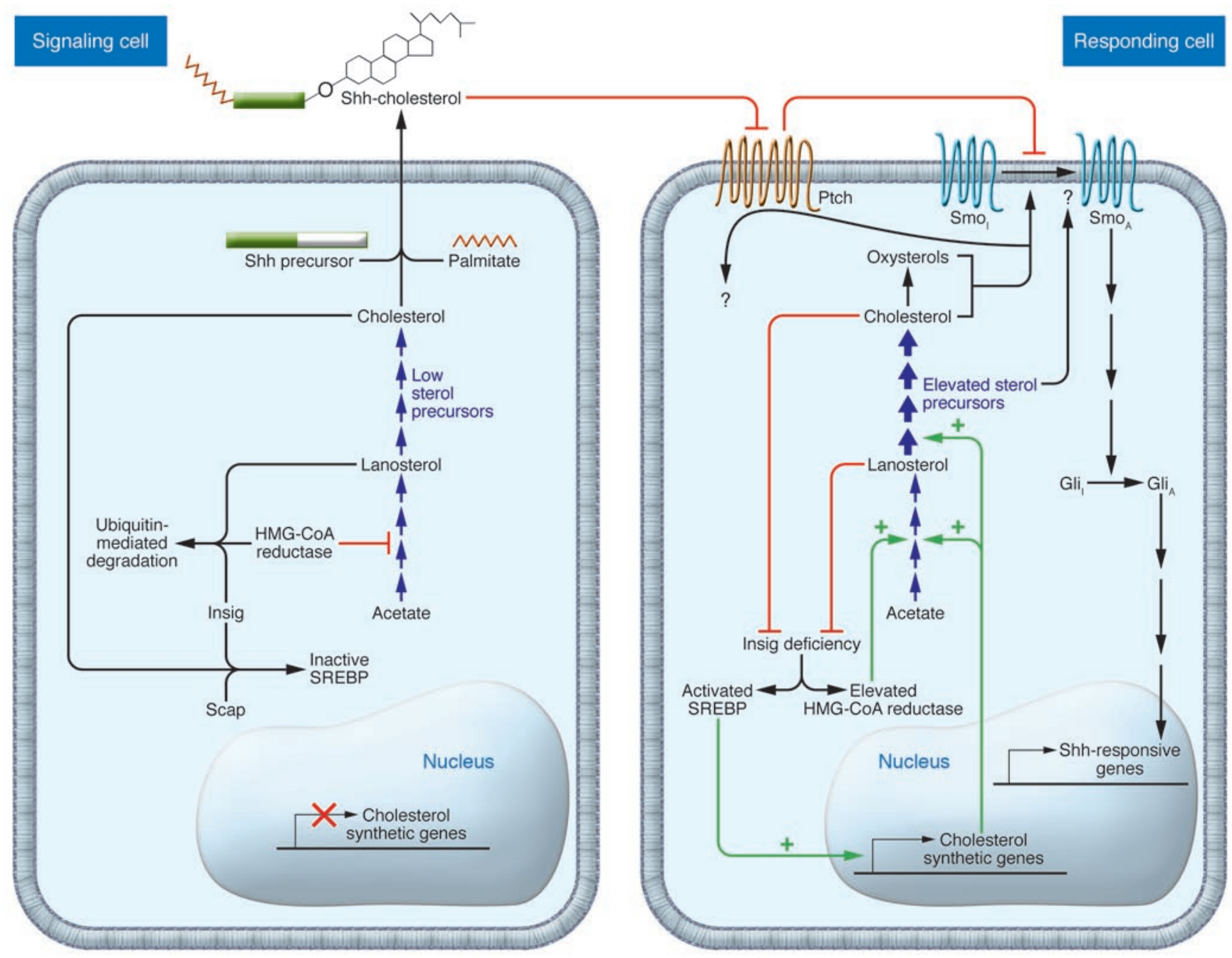

Figure 2

Insig regulation of cholesterol synthesis and Shh signaling. When cholesterol levels are sufficient, Insig proteins regulate cholesterol synthesis by preventing the activation of SREBPs and promoting the degradation of HMG-CoA reductase (left). Cholesterol synthesis becomes dysregulated in Insig-DKO embryos (right). Even when cholesterol levels are elevated, activated SREBPs continue to promote the transcription of cholesterol synthetic genes, and HMG-CoA reductase activity remains high. This results in an accumulation of sterol precursors. The Shh signaling pathway is sensitive to perturbation of cholesterol homeostasis. In the signaling cell (left), Shh protein is modified by the addition of cholesterol. In the responding cell (right), Ptch maintains Smo in an inactive state $\left(\mathrm{Smo}_{\mathrm{I}}\right)$, perhaps by preventing sterol binding. Shh binding to Ptch relieves this inhibition. Activation of $\mathrm{Smo}\left(\mathrm{Smo}_{\mathrm{A}}\right)$ promotes transcription of Shh-responsive genes through the Gli family of transcription factors. Adequate cholesterol or oxysterol levels appear to be necessary for Smo activation. Accumulating sterol precursors in the Insig-DKO embryos could, perhaps via a direct interaction with Smo, alter Shh signaling and underlie the genesis of facial clefting reported by Engelking et al. in Insig-DKO mice (6). Gli, inactive Gli; Gli ${ }_{A}$, activated Gli.

Ptch, like Scap and HMG-CoA reductase, contains a sterol-sensing domain. In the absence of Shh, Ptch functions to maintain the signal-transducing protein Smoothened (Smo) in an inactive state. Initial hypotheses predicted that 7-DHC would disrupt the processing of Shh. However, Cooper et al. (15) showed that Shh signaling in SLOS fibroblasts is impaired at the level of Smo, due to decreased membrane sterol levels. This group hypothesized that total sterol levels may affect the balance of active and inactive forms of Smo. Recently, Corcoran and Scott (16) showed that Smo signal transduction is stimulated by either cholesterol or oxysterols, possibly by binding directly to Smo and stabilizing the active form.

It is plausible that altered Shh signaling contributes to the facial clefting found in Insig-DKO embryos. Two potential mechanisms have been proposed. First, Insig proteins could directly interact with Ptch. This would be consistent with the known interaction of Insig with sterol-sensing domain-containing proteins. Second, an accumulating sterol precursor could modulate Smo function. Small molecule modulators, both agonists and antagonists, of the hedgehog signaling pathway have been identified (17). Thus, either a gain or loss of Smo function could be proposed. In the current study, Engelking et al. (6) were able to decrease the frequency and severity of facial clefting in Insig-DKO mice by treating them with lovastatin. Lovastatin is an 
HMG-CoA reductase inhibitor and lowers sterol precursor levels in Insig-DKO embryos. This experiment strongly supports the idea that elevated sterol precursors underlie the facial clefting. Future work will investigate whether this is mediated through an alteration of Shh signaling.

\section{Acknowledgments}

I would like to thank Steven Fliesler for his critical review of this manuscript. I also would like to express my gratitude to my patients and their parents, whose participation allows my group's research to proceed. This work was supported by the intramural program of the National Institute of Child Health and Human Development, NIH.

Address correspondence to: Forbes D. Porter, HDB, NICHD, NIH, Building 10, Room 9D42, 10 Center Dr., Bethesda, Maryland 20892, USA. Phone: (301) 435-4432; Fax: (301) 480-5791; E-mail: fdporter@ mail.nih.gov.
1. Kelley, R.I., and Hennekam, R.C. 2000. The SmithLemli-Opitz syndrome. J. Med. Genet. 37:321-335.

2. Irons, M., Elias, E.R., Salen, G., Tint, G.S., and Batta, A.K. 1993. Defective cholesterol biosynthesis in Smith-Lemli-Opitz syndrome. Lancet. 341:1414.

3. Porter, F.D. 2002. Malformation syndromes due to inborn errors of cholesterol synthesis. J. Clin. Invest. 110:715-724. doi:10.1172/JCI200216386.

4. Shackleton, C., Roitman, E., Guo, L.W., Wilson, W.K., and Porter, F.D. 2002. Identification of 7(8) and $8(9)$ unsaturated adrenal steroid metabolites produced by patients with 7-dehydrosterol-delta7reductase deficiency (Smith-Lemli-Opitz syndrome). J. Steroid Biochem. Mol. Biol. 82:225-232.

5. Wassif, C.A., Yu, J., Cui, J., Porter, F.D., and Javitt, N.B. 2003. 27-Hydroxylation of 7- and 8-dehydrocholesterol in Smith-Lemli-Opitz syndrome: a novel metabolic pathway. Steroids. 68:497-502.

6. Engelking, L.J., et al. 2006. Severe facial clefting in Insig-deficient mouse embryos caused by sterol accumulation and reversed by lovastatin. J. Clin. Invest. 116:2356-2365. doi:10.1172/JCI28988.

7. Goldstein, J.L., DeBose-Boyd, R.A., and Brown, M.S. 2006. Protein sensors for membrane sterols. Cell. 124:35-46.

8. Engelking, L.J., et al. 2005. Schoenheimer effect explained - feedback regulation of cholesterol synthesis in mice mediated by Insig proteins. J. Clin. Invest. 115:2489-2498. doi:10.1172/JCI25614.

9. Wassif, C.A., et al. 2001. Biochemical, phenotypic and neurophysiological characterization of a genetic mouse model of RSH/Smith-Lemli-Opitz syndrome. Hum. Mol. Genet. 10:555-564.

10. Krakowiak, P.A., et al. 2003. Lathosterolosis: an inborn error of human and murine cholesterol synthesis due to lathosterol 5-desaturase deficiency. Hum. Mol. Genet. 12:1631-1641.

11. Wechsler, A., et al. 2003. Generation of viable cholesterol-free mice. Science. 302:2087.

12. Porter, J.A., Young, K.E., and Beachy, P.A. 1996. Cholesterol modification of hedgehog signaling proteins in animal development. Science. 274:255-259.

13. Rice, R., et al. 2004. Disruption of Fgf10/Fgfr2bcoordinated epithelial-mesenchymal interactions causes cleft palate. J. Clin. Invest. 113:1692-1700. doi:10.1172/JCI200420384.

14. Lewis, P.M., et al. 2001. Cholesterol modification of sonic hedgehog is required for long-range signaling activity and effective modulation of signaling by Ptc1. Cell. 105:599-612.

15. Cooper, M.K., et al. 2003. A defective response to Hedgehog signaling in disorders of cholesterol biosynthesis. Nat. Genet. 33:508-513.

16. Corcoran, R.B., and Scott, M.P. 2006. Oxysterols stimulate Sonic hedgehog signal transduction and proliferation of medulloblastoma cells. Proc. Natl. Acad. Sci. U. S. A. 103:8408-8413.

17. Frank-Kamenetsky, M., et al. 2002. Small-molecule modulators of Hedgehog signaling: identification and characterization of Smoothened agonists and antagonists. J. Biol. 1:10.

\section{Adaptive human regulatory T cells: myth or reality?}

\section{Lucienne Chatenoud and Jean-François Bach}

Université René Descartes Paris 5, INSERM U580, Hôpital Necker-Enfants Malades, Paris, France.

\begin{abstract}
It is now well established that a distinct subset of $T$ lymphocytes is essential for downregulating immune responses to both endogenous (self) and exogenous antigens. These Tregs are $\mathrm{CD}^{+}$and express high levels of CD25 (the $\alpha$ chain of the IL-2 receptor) and the transcription factor Foxp3. The mechanisms determining the lifespan, homeostasis, and in vivo generation of these Tregs are still ill defined. A study by Vukmanovic-Stejic et al. in this issue of the JCI shows that in humans, Tregs are present throughout life but that despite their high throughput, they are short lived (see the related article beginning on page 2423 ). It is thus unlikely that all CD4 ${ }^{+} \mathrm{CD} 25^{\mathrm{hi}} \mathrm{Foxp} 3^{+}$ Tregs are generated as a separate lineage in the thymus. The authors propose that during adulthood, Tregs essentially emerge at the periphery from the memory $\mathrm{T}$ cell pool.
\end{abstract}

The existence in humans of naturally occurring $\mathrm{CD} 4^{+} \mathrm{CD} 25^{+}$Tregs is highlighted by the rapid onset of a severe polyautoimmune syndrome in children genetically deficient for the transcription factor Foxp3, a lineage marker for this Treg subset. Based on this observation in humans and also on

Conflict of interest: The authors have declared that no conflict of interest exists.

Citation for this article: J. Clin. Invest. 116:2325-2327 (2006). doi:10.1172/JCI29748. compelling experimental evidence obtained from the study of mice (1-4), it is postulated and generally accepted that Tregs are responsible for the control of autoreactive $\mathrm{T}$ cells, which react aggressively toward the host's tissues and trigger autoimmune disease in the absence of effective $T$ cell-mediated regulation.

A growing number of studies indicate that Tregs also play a significant role in downregulating the immune response to a variety of nonself antigens, such as micro- bial antigens, tumor antigens, and alloantigens (5-7). Some confusion persists, however, concerning the precise definition of the cell phenotype, functional capacity, and fine antigen specificity of the Tregs participating in these diverse contexts. The situation is further complicated by the fact that some studies argue for the participation of selective and distinct types of Tregs under each of these different conditions (8-13). In contrast, other studies adopt a more unifying view and propose that a single population of $\mathrm{T}$ cells, namely, thymus-derived $\mathrm{CD} 4{ }^{+} \mathrm{CD} 25^{\text {hi }} \mathrm{Foxp}^{+} \mathrm{T}$ cells, gives rise to Tregs controlling the various immune responses (5).

It is apparent that these latter $\mathrm{CD}_{4}^{+}$ $\mathrm{CD} 25^{\text {hiFoxp }} 3^{+} \mathrm{T}$ cells have a predominant role in controlling autoimmune responses in normal individuals in a phenomenon known as physiological tolerance. It is probably an oversimplification, however, to infer that these cells play a role in the control of all other immune responses, especially since effective immune regulation is 